\title{
Smart Wheelchair with Voice Control for Physically Challenged People
}

\author{
Md Abdullah Al Rakib, Salah Uddin, Md. Moklesur Rahman, Shantanu Chakraborty, \\ Md. Ashiqur Rahman, and Fysol Ibna Abbas
}

\begin{abstract}
A wheel chair is a mechanically operated device that allows the user to move about independently. This minimizes the user's personal effort and force required to move the wheelchair wheels. Furthermore, it allows visually or physically handicapped people to go from one location to another. Voice commands and button controls can be used to operate wheelchairs. In recent years, there has been a lot of interest in smart wheelchairs. These gadgets are very handy while traveling from one location to another. The devices can also be utilized in nursing homes where the elderly have difficulties moving about. For individuals who have lost their mobility, the gadgets are a godsend. Different types of smart wheelchairs have been created in the past, but new generations of wheelchairs are being developed and utilized that incorporate the use of artificial intelligence and therefore leave the user with a little to tamper with. The project also intends to develop a comparable wheel chair that has some intelligence and so assists the user in his or her mobility.
\end{abstract}

Key words - Arduino Mega, Bluetooth Module, Smart Wheel Chair, Voice Control, Motor Driver Module.

\section{INTRODUCTION}

People with arm and hand impairments find it difficult to utilize a standard wheelchair since their hands are incapable of operating it and cannot move it in any direction. As a result, a voice operated wheel chair is designed to overcome such people's difficulties and enable them to manage the wheelchair. The wheelchair will be controlled by voice instructions using the input provided. The Arduino will handle all of the user's desired directives. Each direction's instructions are written in the form of a program in the Arduino itself. The unilateral mic, which will be positioned according to the user's comfort, will provide spoken commands to the wheelchair. The HC05 Bluetooth module will do speech recognition. Arduino then receives the output from this module [1]. The Arduino's pre-written algorithms assist Arduino in converting these vocal commands into significant output, and the wheelchair will move appropriately. People will gain independence by using a wheelchair control system. The wheelchair control system makes use of a speech recognition technology to trigger and control all of its motions. The technology allows users to

Submitted on October 12, 2021.

Published on Decemember 03, 2021.

Md Abdullah Al Rakib, Dept. of Electrical and Electronic Engineering, City University, Bangladesh; Salah Uddin, Dept. of Electrical and Electronic Engineering, Feni University, Bangladesh; Faculty of Science, University of Dhaka, Bangladesh.

Md. Moklesur Rahman, Faculty of Science, University of Dhaka, Bangladesh.

Shantanu Chakraborty, College of Science \& Mathematics, Valdosta State University, Georgia; Faculty of Science, University of Dhaka, Bangladesh. operate the wheelchair by just speaking into the wheelchair's microphone [2]. The fundamental movement functions are forward and reverse motion, left and right turns, and stop. The spoken words are sent to the speech recognition processor via a flexible microphone that can be bent to the user's specifications. Many physically handicapped people are unable to move any limbs below the neck. As a result, manual and even joystick-controlled wheelchairs are out of the question for these individuals. As a result, the development of voice-activated wheelchairs will answer the question of quadriplegic patients' movement and make them independent of mobility [3]. In our project, we have both voice command and switch control. In this case, we utilized Arduino as a programming device. Where $\mathrm{c}$ has used programming to better the project. The major goal of this project is to Wheel Chair System Project for the main aim of creating a smart system so that we may reap numerous benefits from a single project. These are utilizing voice command or switching for wheel chair control, which sick patients or elderly people can easily operate; the goal is to build a contemporary one primarily for sick patients or the elderly. There are numerous advantages to such a system, including the following: it reduces human efforts, it is beneficial to physically disabled people who are unable to operate home appliances with their hands, it will help to save energy to some extent, because some people are too lazy to go and switch off the appliances manually, it is simple to use for those who have tried it and do not need to operate the home appliance manually by hand, and it reduces risk [4]. The suggested system has several drawbacks as well, such as the fact that it requires an additional supply to operate the model and that the module only recognizes the inserted voice and button. This type minimizes the amount of physical effort required to acquire and identify the command for controlling the mobility of a wheelchair. The given commands can be used to control the wheelchair's speed and direction. Thus, all that is required to ride the wheelchair is a trained voice [5]. Aside from that, the development of this project may be done at a low cost. However, certain changes are needed to make this system more dependable. The wheelchair's design might be enhanced by including wireless communication. We can immediately improve the lives of handicapped persons in the community

Md. Ashiqur Rahman, Faculty of Science, Comilla University, Comilla -3506 , Bangladesh.

Fysol Ibna Abbas, Dept. of Electrical and Electronic Engineering, City University, Bangladesh; Faculty of Science, University of Dhaka, Bangladesh; Graduate School of Science, Tokyo Metropolitan University, Japan. (e-mail: fysolibnaabbas@gmail.com). 
by establishing this system. Finally, we believe that this type of device will help to advance wheelchair technology. The intelligent wheelchair's motor drive and control system has been shown [6]. The voice-controlled intelligent wheelchair based on a microcontroller would be more convenient for handicapped individuals. By avoiding collisions with walls, immovable objects, furniture, and other people, the device can also improve the safety of users who use standard joystick-controlled powered wheelchairs [7].

\section{PRototype DESIGN}

Our project is the voice control wheel chair. In this project, we first built a robot chair and then set up numerous systems for sick patients and the elderly. Which may be taken anywhere by using the voice command and button control while seated in the chair. The wheel chair was initially intended to help the sick and aged. The goal of this article is to create a wheelchair that moves in response to the user's orders. This system is controlled by the wheelchair user's voice or button instructions. The technology is completely self-contained, since the user does not require the assistance of another person to move the wheelchair. There are generally five commands and depending on whatever command is provided by the user, the wheelchair will move appropriately [8].

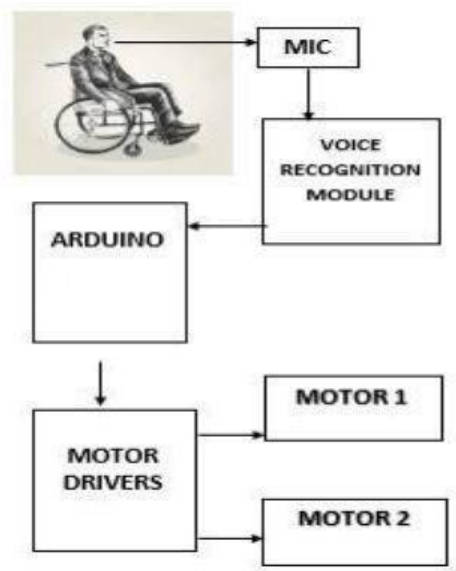

Fig. 1. System Architecture.

In the first stage, the user's vocal instructions are recognized. When a command is acknowledged, it is translated into the corresponding instructions that drive the system. This system is made up of two primary apps and modules: voice recognition and motor driving. The voice recognition is performed via a voice recognition software. The output of this Bluetooth module is sent to Arduino, which drives the motors with a motor driver IC. The unilateral voice recognition app, Bluetooth, motor driver module, Arduino, and motors are used to power the voice-controlled wheelchair. The phone app serves as the system's input. It can take voice commands from the user and ignore other sounds. The mobile app will be positioned according to the user's preference the output is in the form of speech signals, which are sent to the voice recognition software, which serves as a bridge between the Bluetooth module and the Arduino [9][12]. The output from the speech recognition software is then received by the Arduino, which converts it to binary code.
Any language other than binary code is unintelligible to the system. As a result, the produced voice command is translated into machine-readable form. The Arduino Mega is used in this setup. It is linked to motors that allow the wheelchair to be driven anywhere. The wheelchair's mobility is controlled by motors. As a result, motors get input from the Arduino and move in accordance with the kind of instruction. This system has four motors that are linked by a motor driver. The motors may be given four distinct commands: forward, back, left, and right [13]. The wheelchair's mobility is solely dependent on these four instructions. The wheelchair reacts to vocal commands from its user to accomplish any movement's tasks.

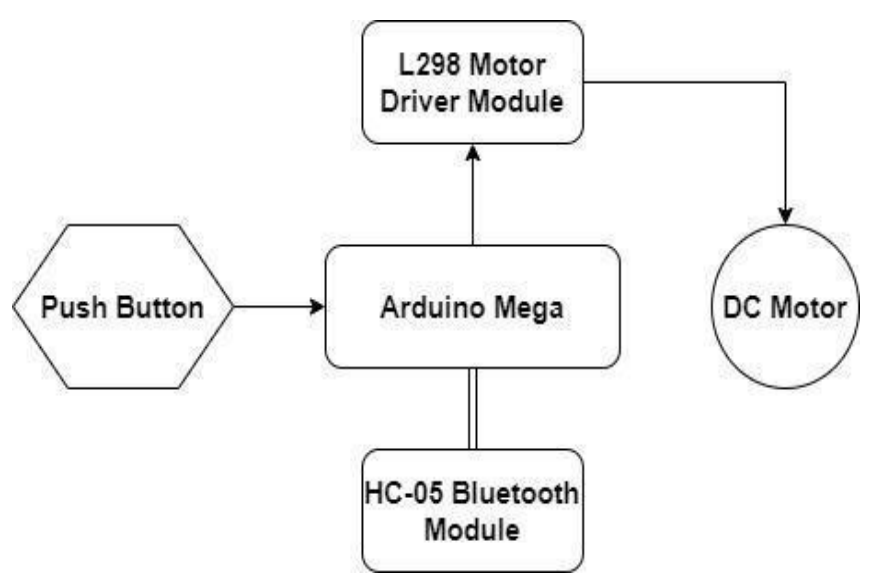

Fig. 2. Block diagram of this work

The fundamental movement functions are forward, left, and right. The speech recognition processor must be taught with the words shouted out by the user who will control the wheelchair in order to recognize the spoken words. In our project, we have both voice command and switch control [14].

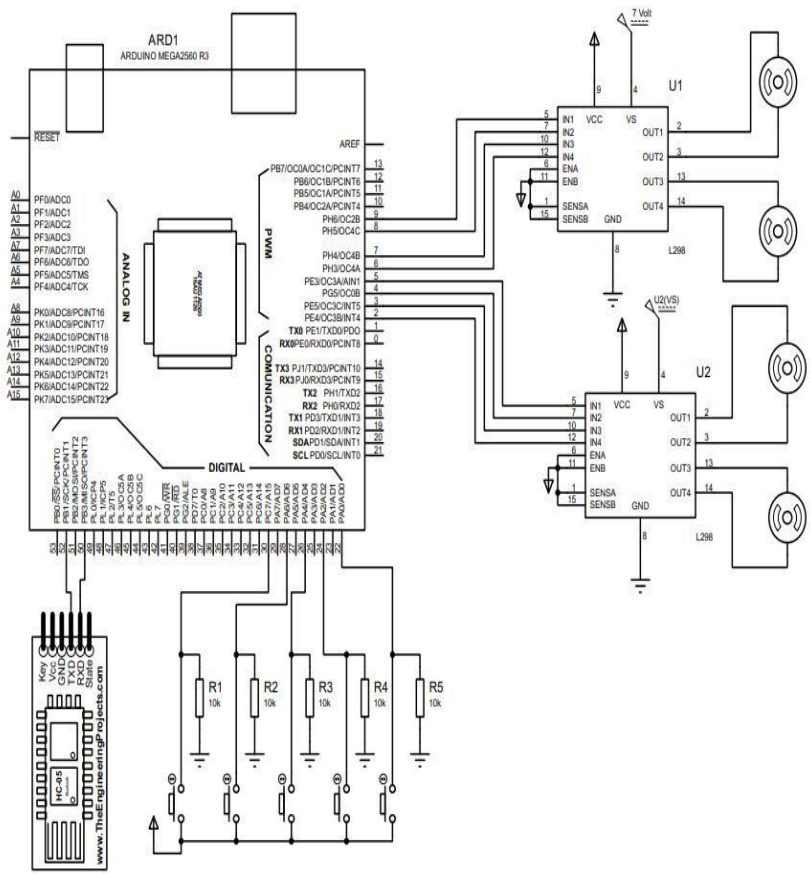

Fig. 3. Circuit diagram of this work.

Serial wireless data transfer is simple using the Bluetooth Module HC-05. Its operational frequency is in the $2.4 \mathrm{GHz}$ ISM frequency range (Industrial, Scientific, and Medical). It 
adheres to the Bluetooth 2.0+EDR specification. The signal broadcast time of different devices in Bluetooth 2.0 is 0.5 seconds.

Seconds' intervals are used to minimize the burden on the Bluetooth chip and conserve more sleeping time for Bluetooth. This module is equipped with a serial interface, which makes it simple to use and shortens the entire design/development cycle [15].

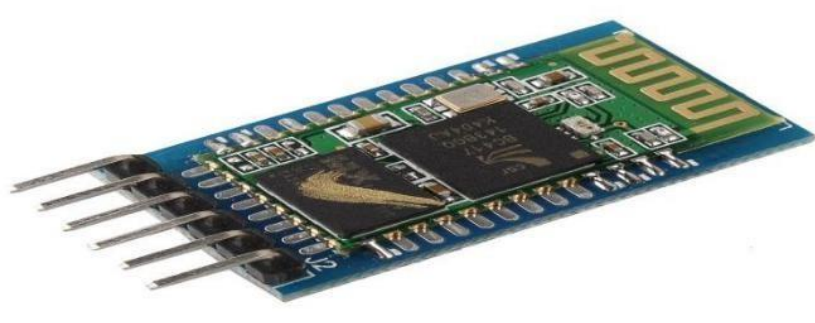

Fig. 4. Bluetooth Module

TABLE I: PIN OUT OF BLuetooth MOdule HC-05

\begin{tabular}{cc}
\hline HC-05 & Sun Founder Uno Board \\
\hline VCC & $5 \mathrm{~V}$ \\
GND & GND \\
TXD & RX0 (pin0) \\
RXD & TX0 (pin1) \\
\hline
\end{tabular}

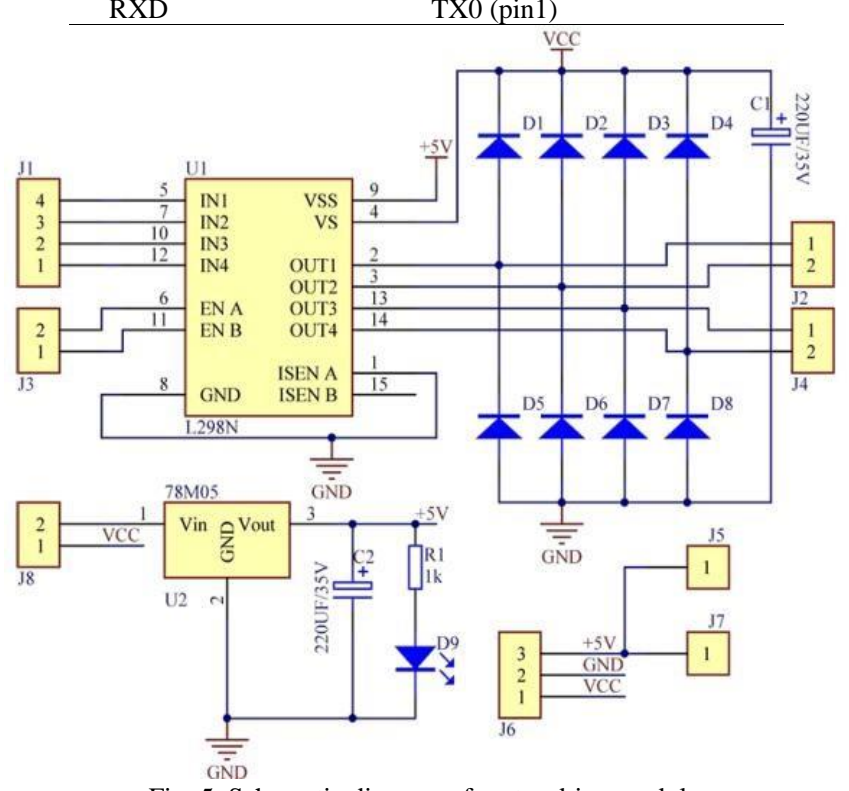

Fig. 5. Schematic diagram of motor drive module.

Installation and configuration of the Arduino software:

1.We downloaded and installed the Arduino software from www.arduino.cc on the computer (This was NOT connected to the PC). After that, we accessed the software file and installed the arduino.exe application. Two program setups are critical and should be examined.

a) On the Arduino software, choose the board to which we wish to connect. The "Arduino Uno" is referred to as the "Arduino / Genuine Uno" in this context [16]-[19].

b) We must select the correct "Serial-Port" to inform the computer to which port the board has been attached. That is only feasible if the USB driver has been properly installed. It may be verified as follows.

Proteus 7.0 is a Virtual System Modeling (VSM) application that integrates circuit simulation, animated components, and microprocessor models to co-simulate whole microcontroller-based systems. This is an ideal tool for engineers who want to test their microcontroller ideas before building a physical prototype in real time. This application allows users to interact with the design by utilizing on-screen indications and/or LED and LCD displays, as well as switches and buttons if they are connected to the PC.

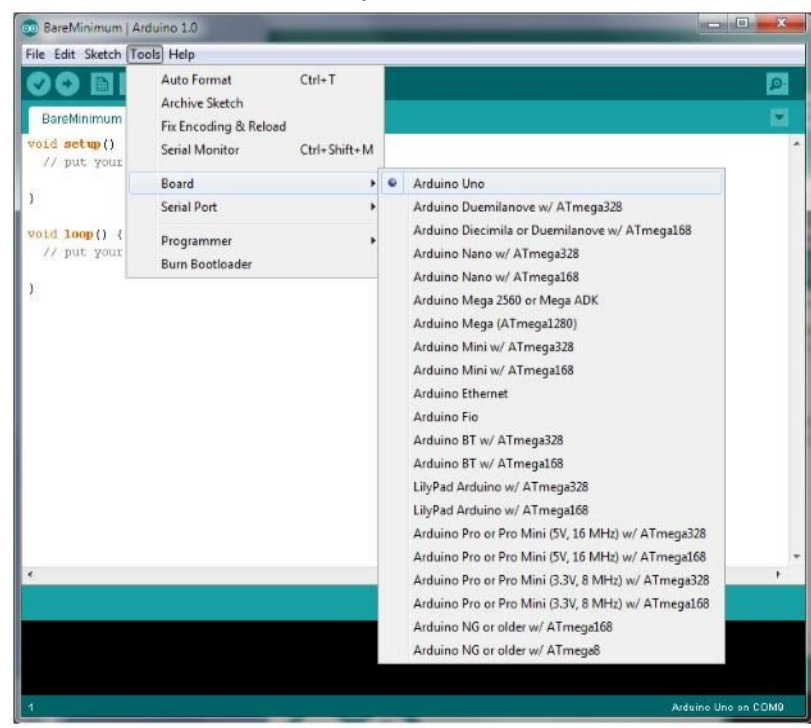

Fig. 6. Program installation process -1 .

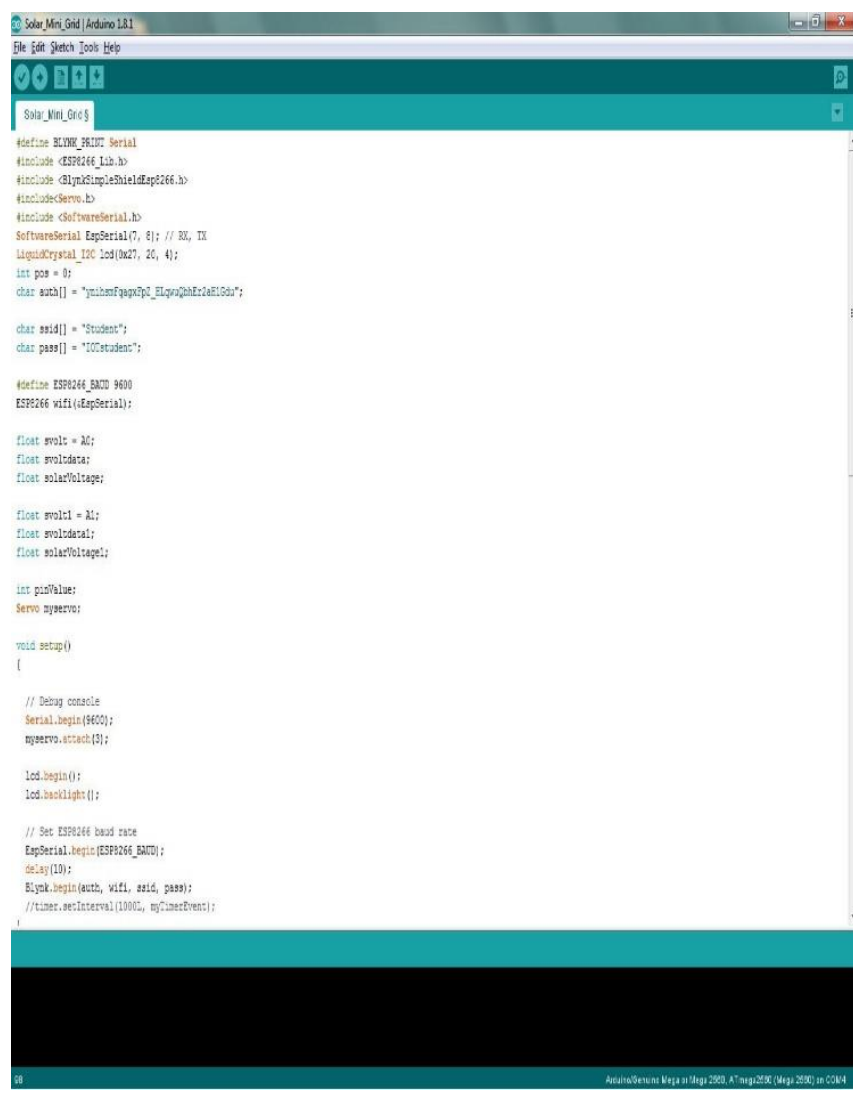

Fig. 7. Program installation process-2.

The Circuit Simulation product, which employs a SPICE3f5 analogue simulator kernel coupled with an eventdriven digital simulator, is one of the core components of Proteus 7.0. It allows users to use any SPICE model from any vendor. For a tidy design prior to hardware implementation, Proteus VSM includes comprehensive debugging tools like as breakpoints, single stepping, and changeable display. In conclusion, Proteus 7.0 is the application to use if you wish 
to mimic the interaction between software running on a microcontroller and any analog or digital electrical device attached to it [20]-[28].

TABLE II: COST ANALYSIS OF THIS WORK

\begin{tabular}{ccccc}
\hline SL & Components Name & Quantity & $\begin{array}{c}\text { Unite } \\
\text { Price }\end{array}$ & $\begin{array}{c}\text { Total Price } \\
\text { (BDT) }\end{array}$ \\
\hline 1 & Arduino Mega & 1 & 920 & 920 \\
2 & Battery & 1 & 1050 & 1050 \\
3 & Motor Driver Module & 2 & 250 & 500 \\
4 & Bluetooth Module & 1 & 320 & 320 \\
5 & Button Switch & 5 & 4 & 20 \\
6 & Car chassis With Gear Motor & & & 800 \\
7 & Others & 1 & 500 & 500 \\
& & & Total Cost & $4,110 \mathrm{tk}$ \\
\hline
\end{tabular}

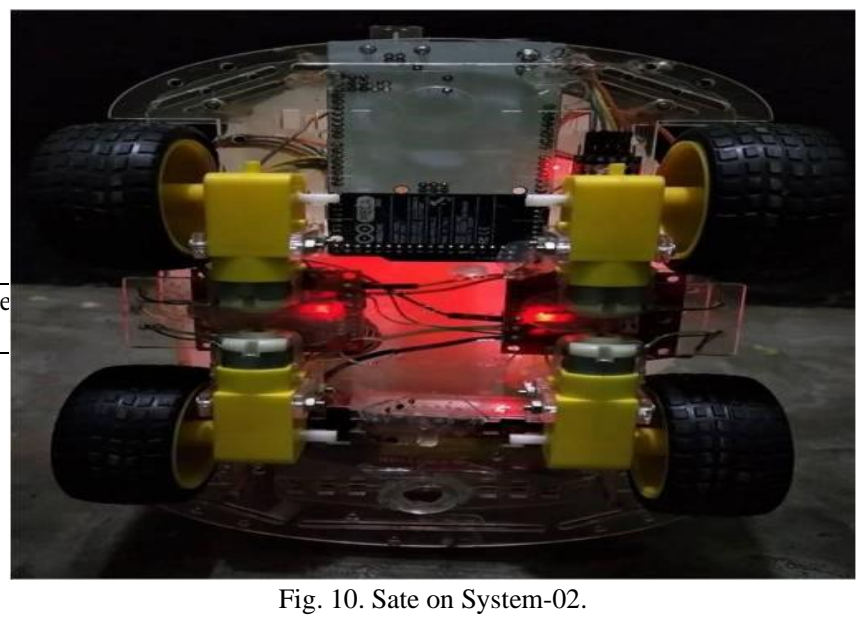

\section{RESULTS}

The project will begin once the power is turned on. The mobile module in the project must be linked to the Bluetooth module. We offer wheelchairs that are controlled by voice using smart phone apps. It may be simply moved to the desired location with the use of a voice command and a switch.

The below image is showing the total system is in OFF mode.

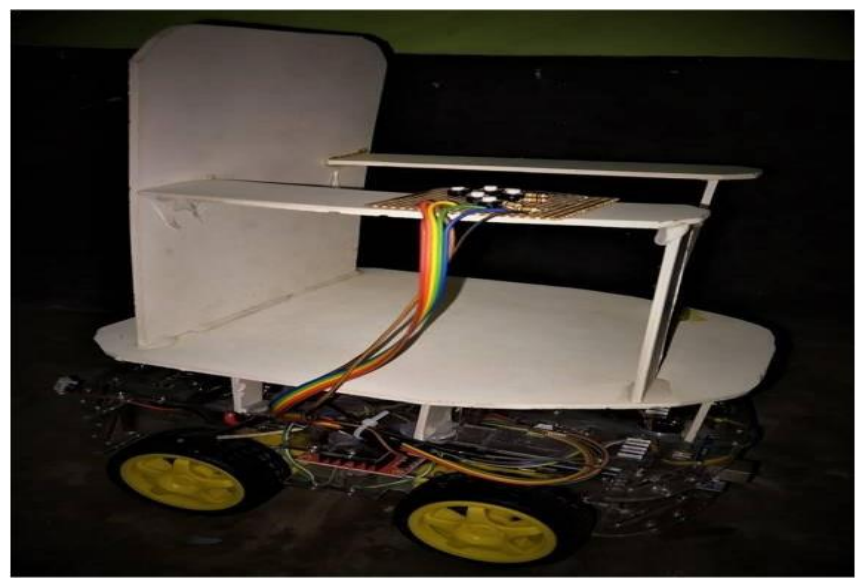

Fig. 8. Hardware part of the prototype.

System operation state $(\mathrm{ON})$ :

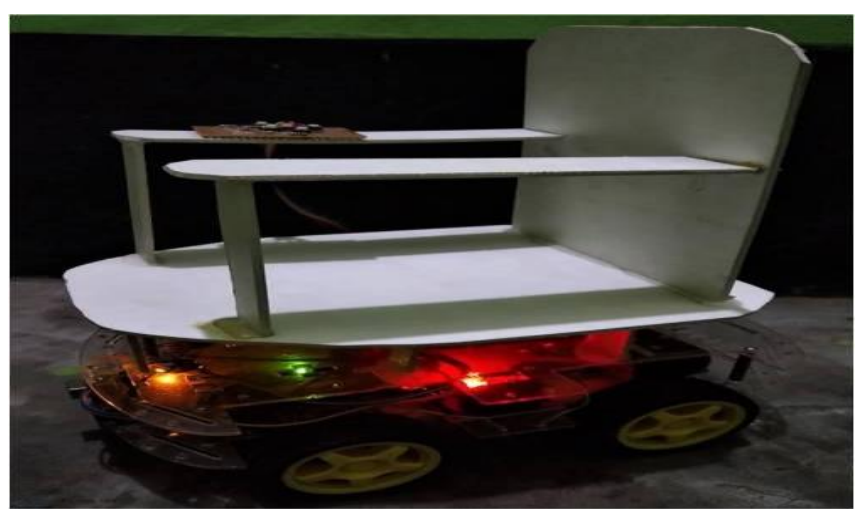

Fig. 9. Sate on System -01.
Our smart wheel chair project uses voice control, which can be accessed via a mobile phone via a set of Android Apps. We've developed the Android applications' functions to change the system control with a single tap.

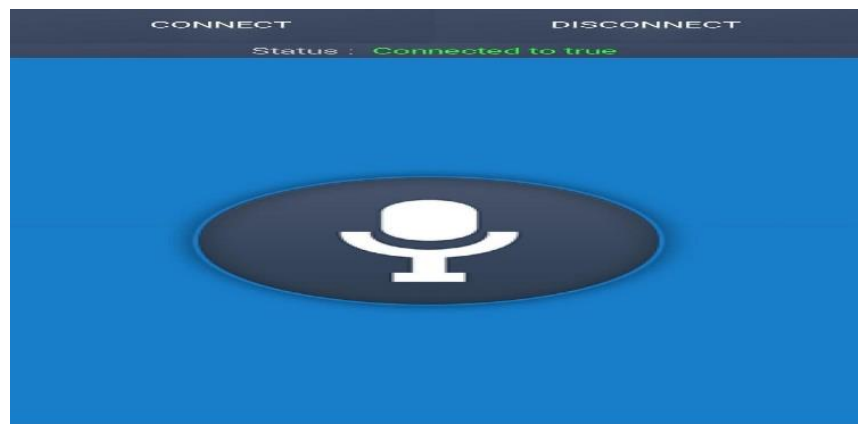

Fig. 11. Arduino Voice Control App.

The advantages of this work are simple-to-control, easy to use, nobody needs a medical specialist to run it, we can use it at home, cheap installation costs, dependable in an emergency.

\section{CONCLUSION}

The design of a smart, powered, voice command, and push button controlled wheelchair utilizing an embedded system is described in this article. The proposed design has a voice activation system for physically disabled people, sick patients, and the elderly. This article depicts a "speech and push button controlled Wheel chair" for physically disabled people, in which the motions of the wheelchair are controlled by a voice command or a switch command. The voice command or switch command is supplied via a Bluetoothenabled cellular device, and the command is transmitted and translated to string by the smart phone app or switch Control for Arduino and is transferred to the Bluetooth Module linked to the Arduino board for Wheelchair control. For example, when the user commands, the chair will move forward, and when he commands, the chair will travel backward, and similarly, "Left," "Right," and "Stop" to rotate it in the left and right directions, respectively, and "Stop" to make it stop. This method was conceived and built to save the patient's money, time, and energy. Even though there is no manufacturing at this level, the project is deemed a success. The above-mentioned problems will be handled, the implementation phase will be completed in order to complete 
the job economically, and this project will be a success because the components utilized are not expensive.

\section{REFERENCES}

[1] Amin MS, et al. Smart Wheelchair-An Implementation of Voice and Android Controlled System. 2021 International Conference on Digital Futures and Transformative Technologies (ICoDT2). IEEE, 2021.

[2] Kataria S, et al. A Novel IOT Based Smart Wheelchair Design for Cerebral Palsy Patients. 2021.

[3] Venkatesan, Mithra, et al. Voice-Controlled Intelligent Wheelchair for Quadriplegics. Computer Communication, Networking and IoT. Springer, Singapore, 2021: 41-51.

[4] Ahmed, Foez, et al., Design and Development of a Smart Wheelchair for the Disabled People. 2021 International Conference on Information and Communication Technology for Sustainable Development (ICICT4SD). IEEE, 2021.

[5] Varghese, Lijo Jacob, et al. Design and Implementation of a Machine Learning Assisted Smart Wheelchair in an IoT Environment. (2021).

[6] Ajay, Mekala, et al. Smart Wheelchair. AI and IoT-Based Intelligent Automation in Robotics, 2021: 271-284.

[7] Tyagi, Anshul, et al. Smart Wheelchair for Disabled People. Research and Reviews: Advancement in Robotics, 2021;4.2.

[8] Chatterjee, Sudipta, and Sahadev Roy. A low-cost assistive wheelchair for handicapped \& elderly people. Ain Shams Engineering Journal 2021.

[9] Basak, Sarnali, et al. Gesture-based Smart Wheelchair for Assisting Physically Challenged People. 2021 International Conference on Computer Communication and Informatics (ICCCI). IEEE, 2021.

[10] Karn, Pratiksha, et al. Smart Wheelchair with SMS Alert and Safety Features.

[11] Ahmad, Shamim, et al. A low SAR in-body antenna for wireless monitoring purpose of pacemaker system. 2019 4th International Conference on Electrical Information and Communication Technology (EICT). IEEE, 2019.

[12] Al Rakib, Md Abdullah, et al. Design and Simulation-Based Parametric Studies of a Compact Ultra-Wide Band Antenna for Wireless Capsule Endoscopy System at Inside Body Environment. International Journal of Electrical and Electronic Engineering \& Telecommunications, 2021.

[13] Al Rakib, Md Abdullah, et al. Energy Harvesting Technology from Human Motion. 2020 2nd International Conference on Advanced Information and Communication Technology (ICAICT). IEEE, 2020.

[14] Mahamud, Md Sadad, et al. Mouchak-An IoT Basted Smart Beekeeping System Using MQTT. 2019 4th International Conference on Robotics and Automation Engineering (ICRAE). IEEE, 2019.

[15] Al Rakib, Md Abdullah, et al., Dry and Wet Waste Segregation and Management System. European Journal of Engineering and Technology Research, 2021;6.5: 129-133.

[16] G. M. Bhuiyan, et al. Local minimum in effective pairpotentials: Pseudopotential theory revisited. Cornell University Library, arXiv preprint arXiv: $1710.07931,2017$.

[17] Mohaiminul Islam, et al. Effects of interionic pair interactions on atomic transport properties of liquid Al, Indian Journal of Physics, pp. 1-10, Springer India, 2021.

Fysol Ibna Abbas, et al. Critical Properties of Segregation for $\mathrm{Al}_{1-\mathrm{x}} \mathrm{Bi}_{\mathrm{x}}$ Liquid Binary Alloys. Journal of the Physical Society of Japan, 2020;89(11):114004.

[18] N. E Dubinin, et al. Effective Wills-Harrison Pair Interaction in Liquid Au. Russian Metallurgy (Metally), 2019; 8:835-837.

[19] G. M. Bhuiyan, et al. Local minimum in pair potentials of polyvalent metals: A limitation of pseudopotential theory. International Journal of Modern Physics B, 2019; 33(7).

[20] Fysol Ibna Abbas, et al. A study of thermodynamics of mixing for $\mathrm{Al}_{1-}$ ${ }_{\mathrm{x}} \mathrm{Sn}_{\mathrm{x}}$ liquid binary alloy, Journal of non-crystalline solids, 2018:481:391-396, Publisher North-Holland.

[21] Fysol Ibna Abbas, et al. Energy of mixing and entropy of mixing for $\mathrm{Cu}_{\mathrm{x}} \mathrm{Al}_{1-\mathrm{x}}$ liquid binary alloys, arXiv preprint arXiv:1607.05827, 2016.

[22] Al Rakib, Md Abdullah, et al. IoT based Controlling of Power Grid. European Journal of Engineering and Technology Research, 2021; 6.6: 54-57.

[23] Al Rakib, Md Abdullah, et al. GSM Based Home Safety and Security System. European Journal of Engineering and Technology Research 2021;6.6: 69-73.

[24] S. Ahmad et al. Design of a Compact Simple Structural Dual-band Patch Antenna for Wireless On-body Medical and Sports Devices, 2021 2nd International Conference on Robotics, Electrical and Signal Processing Techniques (ICREST), IEEE, pp. 577-581, 2021.
[25] Al Rakib, Md Abdullah, et al. An Arduino Based Smart Hand Gloves for Load Control and Physician Notification, 2021 International Conference on Automation, Control and Mechatronics for Industry 4.0 (ACMI), IEEE, p 1-5, (2021).

[27] Al Rakib, Md Abdullah, et al. Low-Cost Pulmonary Ventilator for Patient Monitoring Covid19 Disease. Submitted to European Journal of Engineering and Technology Research (2021).

[28] Al Rakib, Md Abdullah, et al. A Meandered Line Patch Antenna at Low Frequency Range for Early Stage Breast Cancer Detection. Indonesian Journal of Electrical Engineering and Informatics (IJEEI) 2021; 9.3: 662-674.

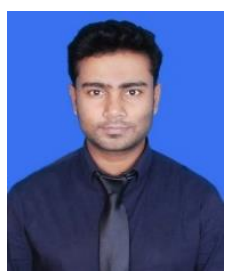

Md Abdullah Al Rakib received Bachelor of Science (B.Sc.) in EEE from American International University-Bangladesh (AIUB). He started his career in September 2018 at City UniversityBangladesh (CUB) as Lecturer in the Dept. of EEE. His research interest is focused on Power, electronics, IoT, and Telecommunication.

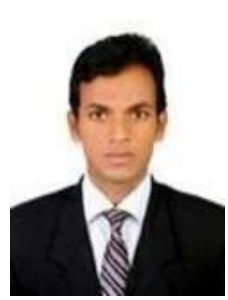

Salah Uddin completed his graduation and postgraduation in Physics and Biomedical physics from University of Dhaka in the year of 2010 and 2015 respectively. Currently he is a lecturer of Physics, in the faculty of EEE in Feni University. His research interest is on material physics, IoT and condensed matter physics.

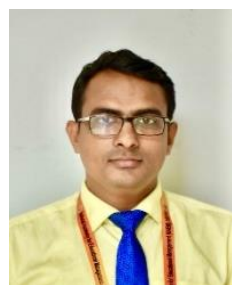

Md. Moklesur Rahman received his M.Sc. in Physics from Dhaka University, Bangladesh in 2012 and B.Sc. in Physics from Dhaka University, Bangladesh in 2011. He joined as the BCS Education cadre in 2016. He is currently working as a Lecturer in the Department of Physics, Shahzadpur Govt. College, Sirajganj,

Bangladesh. He has more than 5 years of experience in teaching and research. His research interests are in Material Physics, IoT and Condensed Matter Physics.

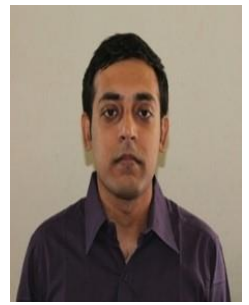

Dr. Shantanu Chakraborty completed his Bachelor and Masters degree from University of Dhaka. He received his $\mathrm{PhD}$ from Florida State University. Currently, he is working as an Assistant Professor of Physics in Valdosta State University. His areas of interest include microwave spectroscopy, optics, material science and IoT.

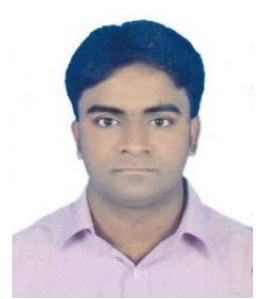

Md. Ashiqur Rahman has completed his undergraduation degree (B.Sc.) \& graduation degree (M.Sc.) in Physics from Department of Physics, University of Dhaka. He is currently doing his $\mathrm{Ph} . \mathrm{D}$. research at Graduate School of Science, Tokyo Metropolitan University at Tokyo prefecture Japan.He served as a Lecturer in the Department of Physics, Comilla University $(\mathrm{CoU})$, Bangladesh from $31^{\text {th }}$ July, 2016 to $30^{\text {th }}$ November, 2018. He promoted as an Assistant Professor in the same University from $1^{\text {st }}$ December 2018. Still Now, he has worked as an Assistant Professor in the Department of Physics, Comilla University (CoU), Bangladesh. His research interests are inorganic nanotube, carbon nanotube, thermoelectric materials, Superconductivity, Nanotechnology and Optics. 


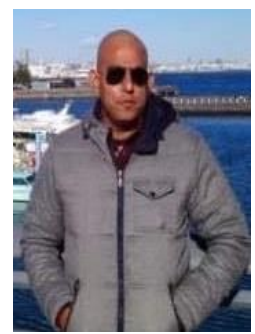

Dr. Fysol Ibna Abbas received his Ph.D. in Material Science from the Department of Theoretical Physics, University of Dhaka, Bangladesh. The worldwide well-known Professor Dr. Arghya Taraphder, (IIT-Indian Institute of Technology Kharagpur, India) \& Professor Dr. David J. Gonzalez (The University of Valladolid, Valladolid province, Spain) were the doctoral thesis reviewer of him from 22 July 2018 to 25 February 2019. Both of them are specialists in Material Science research works. Dr. Abbas completed his M.Sc from the Department of Theoretical Physics (Condensed Matter Research Group) at the University of Dhaka in 2012. From the same university, he completed his B.Sc in Physics (2010). In the Graduate school of science, Tokyo Metropolitan University at Tokyo prefecture, Japan, Dr. Abbas is currently working as an active researcher. In his service career, he served as an Assistant Professor in the Department of Electrical \& Electronic Engineering, City University Bangladesh (CUB) from 2018 to 2021 . He has also served as a faculty member in the MNS Department at BRAC University from 2013 to 2018 Dr. Abbas has more than 12 years of experience in teaching and research. His research interests are Liquid binary alloys, Nuclear Physics (Theory), High entropy alloys (HEA), Thermoelectric materials (TE), Functional materials application, Superconductivity (SC), Photonics, Nanotechnology, Optics, and Solar cell. 